\title{
CGM, Pregnancy, and Remote Monitoring
}

\author{
Sarit Polsky, MD, MPH, and Rachel Garcetti, PA
}

\begin{abstract}
The glycemic goals of pregnancy are very narrow to reduce excess risks for numerous maternal and fetal complications. Continuous glucose monitors (CGMs) may help women achieve glucose goals and reduce hypoglycemia. CGM use has been found to be safe and effective in pregnancies associated with diabetes. CGM use can accurately identify glycemic patterns among women with and without diabetes in pregnancy. The data on the effects of CGM use on maternal and fetal outcomes are conflicting. Using CGMs in conjunction with continuous subcutaneous insulin infusion therapy in pregnancies complicated by diabetes may improve outcomes. There are limitations of CGM use that affect patients in and outside of pregnancy, as well as specific barriers that only affect pregnant women. Of importance, CGM use does not replace standard clinical care, but may be used an adjunctive tool in pregnancy. CGM remote monitoring in pregnancy is an understudied field. In this study, we review the studies on CGM use in pregnancy.
\end{abstract}

Keywords: Continuous glucose monitoring, Pregnancy, Remote monitoring, Gestational diabetes, Preexisting diabetes.

\section{Introduction}

$\mathbf{P}$ REGNANCIES ASSOCIATED WITH diabetes have high maternal and fetal risks, especially if metabolic control is suboptimal. These risks include fetal loss, congenital anomalies, abnormal fetal size (large-for-gestational age [LGA]; small-for-gestational age; macrosomia), preeclampsia/eclampsia, cesarean section, neonatal hypoglycemia, and neonatal hyperbilirubinemia, among others. ${ }^{1-4}$ Therefore, the American Diabetes Association (ADA) recommends tight glycemic control throughout gestation ${ }^{5}$ (Table 1). One tool that may help achieve optimal glycemic control in pregnancy is the continuous glucose monitor (CGM). CGM use improves hemoglobin A1C (A1C) levels in nonpregnant adults and children without increased hypoglycemia. ${ }^{6-8}$ The ADA recommends CGM use in pregnancy for some high-risk women, such as those with hypoglycemia unawareness. ${ }^{2}$ This review aims to (1) highlight studies on CGM use in pregnancy regarding safety, glycemic parameters, and maternal and fetal outcomes and (2) discuss remote monitoring methods of sensor glucose data and limitations of the technology.

\section{Methods}

We conducted a PubMed electronic search using keywords "CGM," "pregnancy," then "remote monitoring." When data on specific platforms were absent, we conducted a secondary search, for example, for "carelink," " glooko," “diasend," and "Tidepool." We further defined searches when appropriate, for example, "dexcom" and "remote" together. We searched bibliographies of studies identified through the Medline (PubMed) inquiry for additional studies and we reexamined relevant articles previously known to the authors for appropriateness of inclusion. Studies were included that examined CGM effects on outcomes (glucose control, maternal, and fetal) in pregnancy. Articles were excluded if they did not present primary data, did not utilize a CGM system, or did not include pregnant women with diabetes.

\section{Safety and Accuracy of CGMs in Pregnancy}

CGM use has been successful, safe, and accurate in detecting glucose levels in pregnancies with diabetes. ${ }^{9-12}$ Chen et al. investigated CGM use in 57 women with gestational diabetes mellitus (GDM) between 24 and 36 weeks of gestation comparing 3-day CGM data with 6-8 daily selfmonitoring of blood glucose (SMBG) measurements. CGM values strongly correlated with SMBG measurements. CGMs better detected high postprandial glucose levels and nocturnal hypoglycemia than SMBG testing. ${ }^{9}$ In a study with eight women (type 1 diabetes [T1D] and GDM) on multiple daily

Barbara Davis Center for Diabetes, University of Colorado Denver, Aurora, Colorado. 
Table 1. American Diabetes Association Target of Glycemic Goals for Pregnant Women with Diabetes ${ }^{5}$

\begin{tabular}{|c|c|c|c|}
\hline \multirow[b]{2}{*}{ Variable } & \multicolumn{3}{|c|}{ Glycemic targets } \\
\hline & Gestational diabetes $^{\mathrm{a}}$ & Preexisting diabetes $^{\mathrm{a}}$ & $\begin{array}{c}\text { Diabetes with significant } \\
\text { hypoglycemia }\end{array}$ \\
\hline $\begin{array}{l}\text { Glycated Hemoglobin } \\
\text { A1C }\end{array}$ & $<6 \%$ & $<6 \%$ & \\
\hline Fasting glucose & $\leq 95 \mathrm{mg} / \mathrm{dL}(5.3 \mathrm{mmol} / \mathrm{L})$ & 60-99 mg/dL (3.3-5.4 mmol/L) & $<105 \mathrm{mg} / \mathrm{dL}(5.8 \mathrm{mmol} / \mathrm{L})$ \\
\hline Postprandial glucose & $\begin{array}{l}1-\mathrm{h}: \leq 140 \mathrm{mg} / \mathrm{dL}(7.8 \mathrm{mmol} / \mathrm{L}) \\
2-\mathrm{h}: \leq 120 \mathrm{mg} / \mathrm{dL}(6.7 \mathrm{mmol} / \mathrm{L})\end{array}$ & $100-129 \mathrm{mg} / \mathrm{dL}(5.4-7.1 \mathrm{mmol} / \mathrm{L})$ & $\begin{array}{l}1 \mathrm{~h}:<155 \mathrm{mg} / \mathrm{dL}(8.6 \mathrm{mmol} / \mathrm{L}) \\
2-\mathrm{h}:<130 \mathrm{mg} / \mathrm{dL}(7.2 \mathrm{mmol} / \mathrm{L})\end{array}$ \\
\hline
\end{tabular}

\footnotetext{
${ }^{a}$ Aim for targets if achievable without significant hypoglycemia.
}

injections (MDIs), CGM use between 24 and 32 weeks of gestation was associated with effective treatment adjustments as undetected hyperglycemia, nocturnal hypoglycemia, and mean daily glucose levels were all reduced. ${ }^{10}$ Results were consistent in both women with T1D and GDM. Kerssen and colleagues found that CGM values were comparable with SMBG values $(93.8 \%$ of data were in the clinically acceptable zone of the Clarke error grid analysis, EGA) among 15 pregnant women with T1D. CGM use in pregnancy was well tolerated. $^{11}$

A study with 12 women with T1D at high risk for severe hypoglycemia used CGMs from 10 to 20 weeks of gestation. Severe hypoglycemia incidence was decreased from pre- to post-CGM initiation. CGM use may have reduced severe hypoglycemic events later in pregnancy. ${ }^{12}$ Buhling et al. measured rates and duration of hyperglycemia with CGMs or SMBG in nonpregnant and pregnant women (no diabetes, impaired glucose tolerance, or GDM). CGMs identified more episodes of sustained hyperglycemia in GDM compared with SMBG alone. CGMs better differentiated between groups compared with SMBG alone. ${ }^{13}$ More recent studies have not reported significant concerns regarding safety or accuracy of CGM therapy. Thus, in pregnancies associated with diabetes, CGMs with SMBG may be superior to SMBG testing alone.

\section{CGM Pattern Recognition in Pregnancy}

Numerous studies compared CGM glycemic patterns among women with abnormal glucose metabolism in pregnancy with those of women without diabetes in and outside of pregnancy. In 41 pregnant women with T1D, CGM wear for 3 days detected periods of hyperglycemia and hypoglycemia that were missed with SMBG and providers often changed treatment based on CGM profiles. ${ }^{14} \mathrm{~A}$ study in women with T1D, diet-controlled GDM, and insulin-controlled GDM wearing a CGM for 3 days found that within 240 min after a meal, time to peak postprandial glucose level was similar between groups (82-93 $\mathrm{min}$ ), but varied between individuals. About half the subjects did not reach preprandial glucose levels $3 \mathrm{~h}$ after meal consumption. ${ }^{15}$ Other studies corroborated the within-day glucose variation seen with CGM technology that may be missed if relying on SMBG or A1C alone. $^{16-18}$

One study examined postprandial glucose excursions with CGM and SMBG values for 3 days in pregnant women with normal glucose tolerance (NGT, $n=36$ ) and with GDM or T1D $(n=17)$. Time to peak postprandial glucose was similar between groups $(82 \pm 18 \mathrm{~min}$ for NGT and $74 \pm 23 \mathrm{~min}$ for
DM). CGM values were slightly higher among women with diabetes, significantly so at 120 and 135 min postprandially. Differences in 60 to 150 min postprandial glucose levels were significantly associated with mode of delivery, infant birth weight percentile, and/or diabetes-associated complications. ${ }^{19}$ A study looking at CGM profiles for 3-7 days in 17 pregnant women with diabetes compared profiles with women without diabetes ( 1 of 2 was pregnant). Women treated with insulin (GDM or overt DM) had more postprandial hyperglycemia and glycemic variability (measured by standard deviation, SD, and mean amplitude of glycemic excursions, MAGE). ${ }^{20}$

Mazze et al. compared CGM profiles for $\geq 3$ days in the third trimester in 82 women with GDM, pre-existing DM, and NGT with 21 nonpregnant women with NGT. Women without diabetes had narrow glucose profiles in pregnancy. There was a $20 \%$ difference in diurnal glucose patterns between pregnant and nonpregnant women. Glucose variability was highest with pregestational diabetes compared with GDM. ${ }^{21}$ In one study, 50 women with GDM, T1D, and NGT wore CGMs for 2 days each trimester. Multiple indicators of glucose variability were higher, worse, and correlated more strongly with each other for women with T1D. ${ }^{22}$

CGMs have been utilized in special situations in pregnancy, such as pregnancy after Roux-en-Y gastric bypass, ${ }^{23}$ obese pregnancies, ${ }^{24}$ masked (asymptomatic) hypoglycemia in insulin-treated GDM, ${ }^{25}$ and exercise with T1D. ${ }^{26}$ Three of the abovementioned studies showed more or similar rates of hypoglycemia among pregnancies with NGT compared with other groups. $^{21,22,25}$ Therefore, CGMs have elucidated glucose variability and glycemic patterns in pregnancies with and without diabetes and shown that women with T1D and insulin-requiring GDM have more glycemic variability compared with those with diet-controlled GDM. ${ }^{15,20,21}$

\section{Randomized Controlled Trials of CGM Use in Pregnancy}

Some randomized controlled trials (RCTs) have examined CGM use in pregnancy (Table 2). In one study, only $31 \%$ (5/16) of CGM users who met criteria for antihyperglycemic medication would have been identified with SMBG values alone. ${ }^{27}$ Murphy et al. randomized women with T1D and type 2 diabetes (T2D) to standard care or intermittent CGM use between 8 and 32 weeks of gestation. CGMs were worn for a week every 4-6 weeks with data reviewed after each session. A1C was significantly lower in the CGM users between 32 and 36 weeks of gestation. ${ }^{28}$ At 36 weeks of gestation, despite a mean $\mathrm{A} 1 \mathrm{C}$ under $6 \%$, time spent with sensor glucose levels 


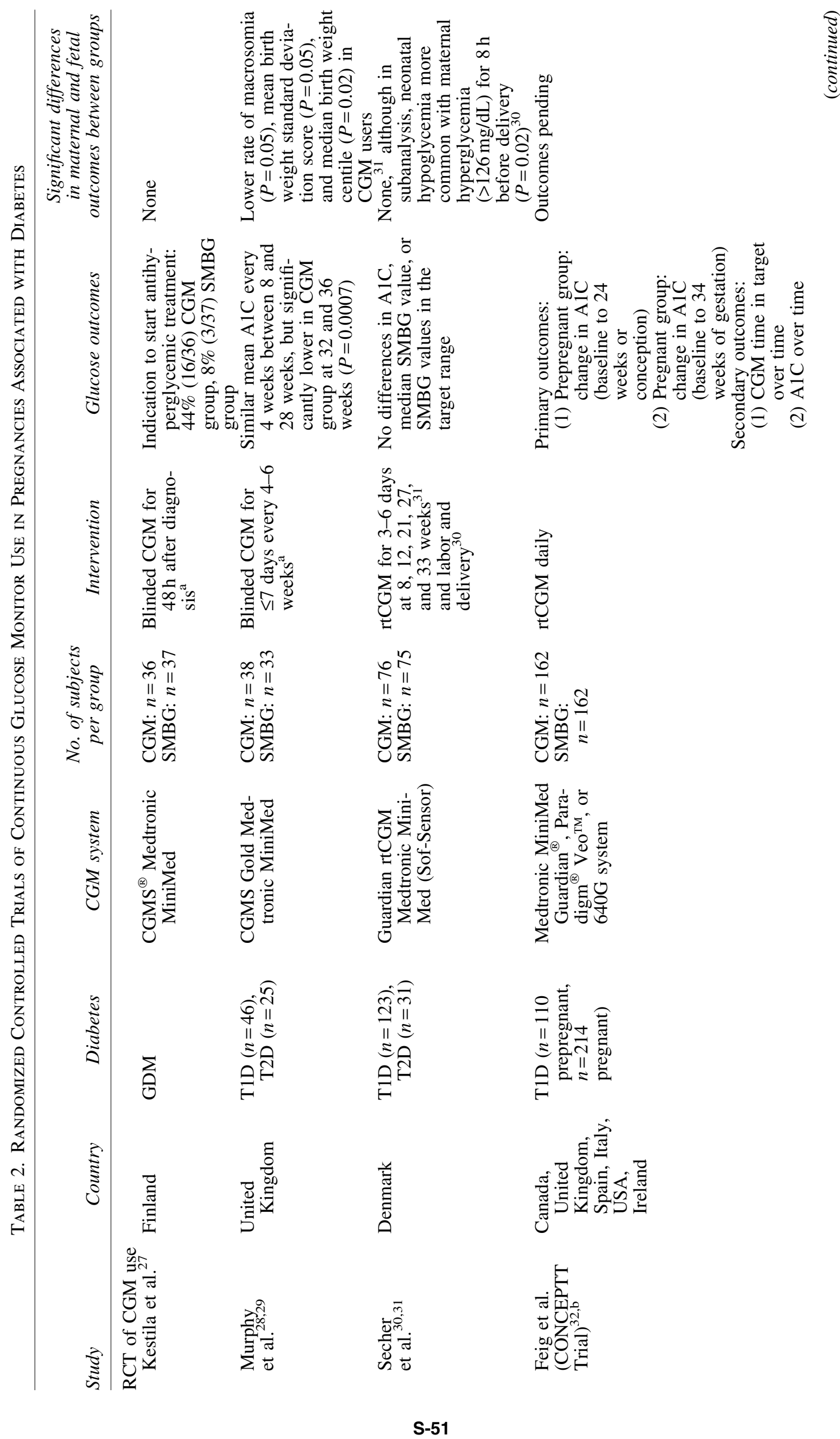




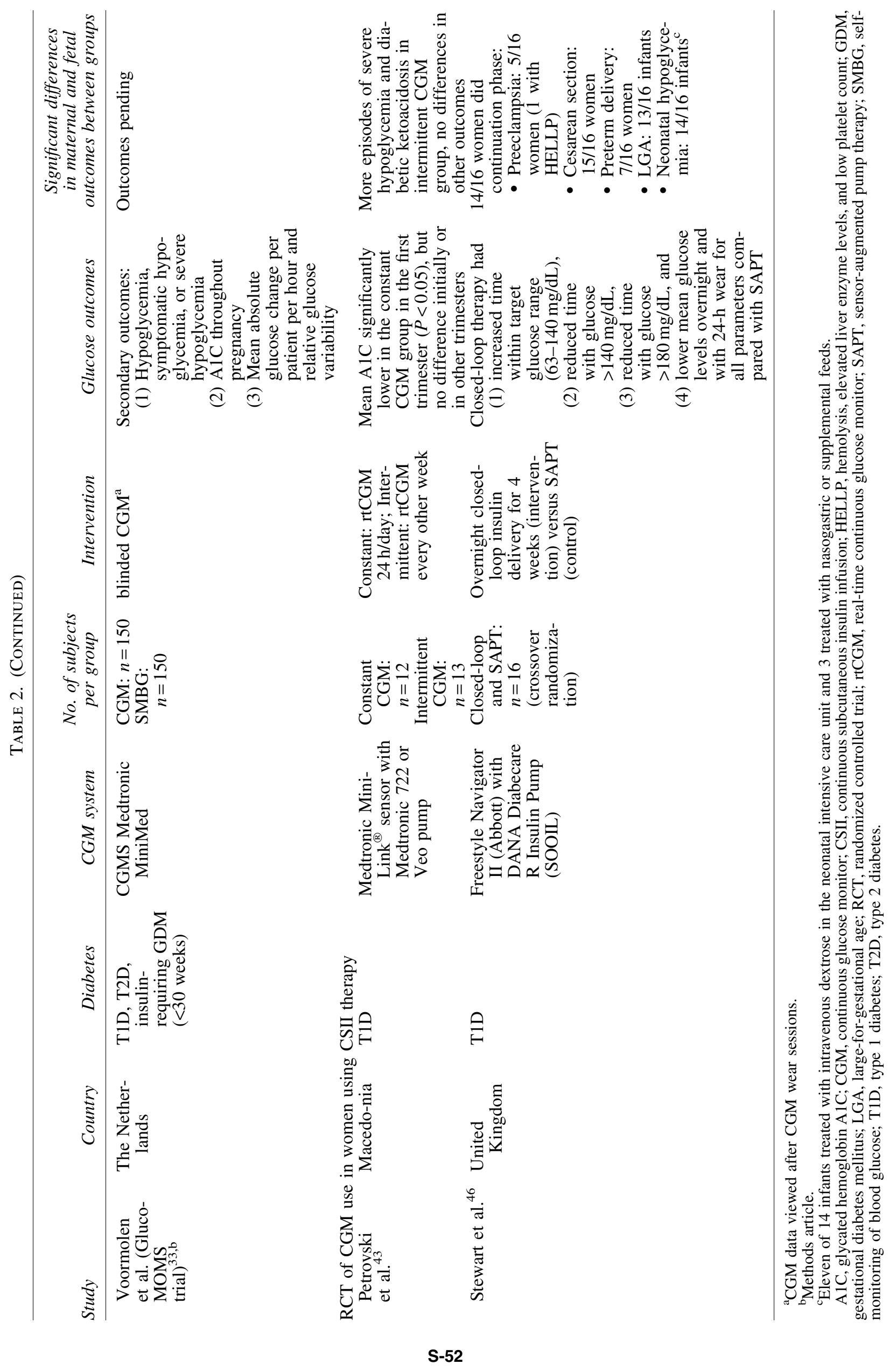


$>140 \mathrm{mg} / \mathrm{dL}$ was $\geq 8 \mathrm{~h} /$ day in women with T1D and $\sim 3 \mathrm{~h} /$ day in women with T2D in each trimester. ${ }^{29}$

The Danish study of intermittent real-time CGM (rtCGM) found no differences in outcomes between groups; however, only $64 \%$ (49/76) in the rtCGM group followed the protocol. $^{30,31}$ Taken together, these studies suggest that adjunctive CGM use may help make decisions about therapy, but improvement of long-term glucose control and outcomes may require more than just intermittent use.

Two rtCGM RCTs published their methods, completed their studies, and have adequate power to address the effectiveness of CGM therapy in pregnancy. The CONCEPTT trial randomized 324 women with T1D to standard care or SMBG with daily CGM use. ${ }^{32}$ The GlucoMOMS trial randomized 300 women with diabetes (T1D or T2D before 16 weeks of gestation and GDM) to standard care or SMBG testing with masked CGM. ${ }^{33}$ These RCTs (CONCEPTT and GlucoMOMS) will give more definitive data on the efficacy of CGM therapy on maternal glucose control and maternal and fetal outcomes.

\section{CGM Use and Maternal and Fetal Outcomes}

CGMs may affect outcomes of pregnancies complicated by diabetes (Table 3 ). Neonatal hypoglycemia is a frequent complication in infants of mothers with diabetes and can have lasting effects on children. ${ }^{34}$ Two studies looked at effects of maternal glucose levels in insulin-treated women during labor and delivery ( 2 to $8 \mathrm{~h}$ before birth) and resultant neonatal hypoglycemia. Stenninger et al. found that multiple glycemic indices correlated positively with the need for neonatal intravenous glucose infusions. ${ }^{34}$ In Secher's study (Table 2), $45 \%$ (27/60) of women in the CGM group were compared with $100 \%$ (59/59) in the control group. Among women in the CGM arm, 10 infants developed hypoglycemia compared with 27 in the non-CGM group (37\% vs. $46 \%$, respectively; $P=0.45){ }^{30}$ They found that maternal hyperglycemia before delivery was correlated with neonatal hypoglycemia.

Glycemic variability has been investigated. One study used blinded 3-day CGM recordings with SMBG data at 26-28 weeks of gestation to measure maternal glucose excursions (24-h magnitude and duration of glucose excursions defined as glycemia index [GI]). GI correlated with fetal growth in all pregnancy populations, especially for GI $>130$. The magnitude of glucose excursions was easier to quantify using CGM and would be underestimated by SMBG alone. ${ }^{35}$ In a study with 340 GDM patients, the women in the CGM group had less glucose variability and better glycemic control. Women using CGM had significantly lower rates of preeclampsia and cesarean sections versus those using SMBG alone. Infants of mothers using CGM had significantly better outcomes compared with SMBG infants. ${ }^{36}$

Macrosomia has been examined in multiple studies. Dalfra et al. found that macrosomia was linked to maternal glycemic variability and hyperglycemia in diabetic pregnancies. ${ }^{37}$ Infants born to mothers intermittently using CGMs had lower birth weights, decreased birth weight percentiles, and a lower risk of macrosomia (odds ratio $0.36,95 \%$ confidence interval 0.13 to 0.98$)^{28}$ (Table 2).

LGA, defined as birth weight $\geq 90$ th percentile for sex and gestational age, affects $50 \%$ of infants of mothers with diabetes, even with clinically well-controlled diabetes. ${ }^{38}$ Law et al. used CGMs in 117 pregnancies complicated by preexisting diabetes to examine the impact of temporal glucose variations on fetal growth. Fifty-four infants (46\%) developed LGA. Mean A1C levels were well controlled. There was no significant difference in maternal A1C levels between mothers with LGA infants verses those without. CGM data identified times of day that hyperglycemia impacted fetal growth more and trimester-specific diurnal glucose differences in women with LGA infants. ${ }^{38}$ Overall, while CGM use with SMBG has been evaluated throughout pregnancies in numerous small studies, it remains unclear whether it improves pregnancy outcomes.

\section{Sensor-Augmented Pump Therapy in Pregnancy}

Sensor-augmented pump therapy (SAPT), simultaneous use of continuous subcutaneous insulin infusion (CSII) therapy with rtCGM, improves glucose control and A1C over MDI alone or CSII use alone in nonpregnant populations. ${ }^{39-41}$ Although studies are sparse, some have investigated SAPT use in pregnancies associated with diabetes (Table 2).

In one retrospective multicenter study, women with T1D using CSII therapy alone $(n=47)$ were compared with those who self-selected CSII therapy with rtCGM $\geq 3$ times/month $(n=18)$ during pregnancy and delivery. Both groups had stable peripartum glycemic values, but mean capillary blood glucose levels were significantly lower in the rtCGM group throughout labor compared with CSII alone. ${ }^{42}$ Petrovski et al. randomized 25 women with T1D using CSII therapy to constant or intermittent rtCGM use throughout gestation. A1C levels improved in both groups throughout pregnancy, but were significantly lower in early gestation in the constant CGM group. ${ }^{43}$

Finally, closed-loop insulin delivery employs feedback from rtCGM into mathematical algorithms inside insulin pumps to adjust insulin delivery to maintain euglycemia. Murphy et al. conducted three studies on hybrid closed-loop insulin delivery systems in pregnant women with T1D wherein women bolused for carbohydrates and insulin delivery was otherwise adjusted by the systems. The first study used CGMs for $24 \mathrm{~h}$ during early (12-16 weeks) and late gestation (28-32 weeks) in 10 women. Plasma glucose measurements every 15 min showed that time in the target range $(63-140 \mathrm{mg} / \mathrm{dL})$ overnight was $84 \%$ in early and $100 \%$ in late gestation $(0 \%$ hypoglycemia at each time point), but in the postprandial period was reduced (dinner: 68\%-77\%; breakfast: 47\%-59\%). ${ }^{44}$ Subsequently, 12 women were randomized to closed-loop or CSII therapy during daily activities and exercise for $24 \mathrm{~h}$, then crossed over to the alternate therapy. Overall, time in the plasma glucose target range was $81 \%$ during both sessions, while time in hypoglycemia $(\leq 45 \mathrm{mg} / \mathrm{dL})$ was lower with closed-loop therapy ( $0 \%$ closed loop vs. $0.3 \%$ CSII, $P=0.04)$. Nocturnal time in the CGM glucose target range was significantly higher in closed loop. ${ }^{45}$ The FreeStyle Navigator sensor used in these studies was found to have a MARD (mean absolute relative difference) of $11.4 \%$ and Clarke error grid analysis in clinically acceptable zones. ${ }^{44}$ Closed-loop insulin delivery systems appear safe in pregnancy. ${ }^{44,45}$

The last study was a crossover trial wherein 16 pregnant women were randomized to 4 weeks of closed-loop therapy or SAPT with a 2-week washout between sessions. Overnight 


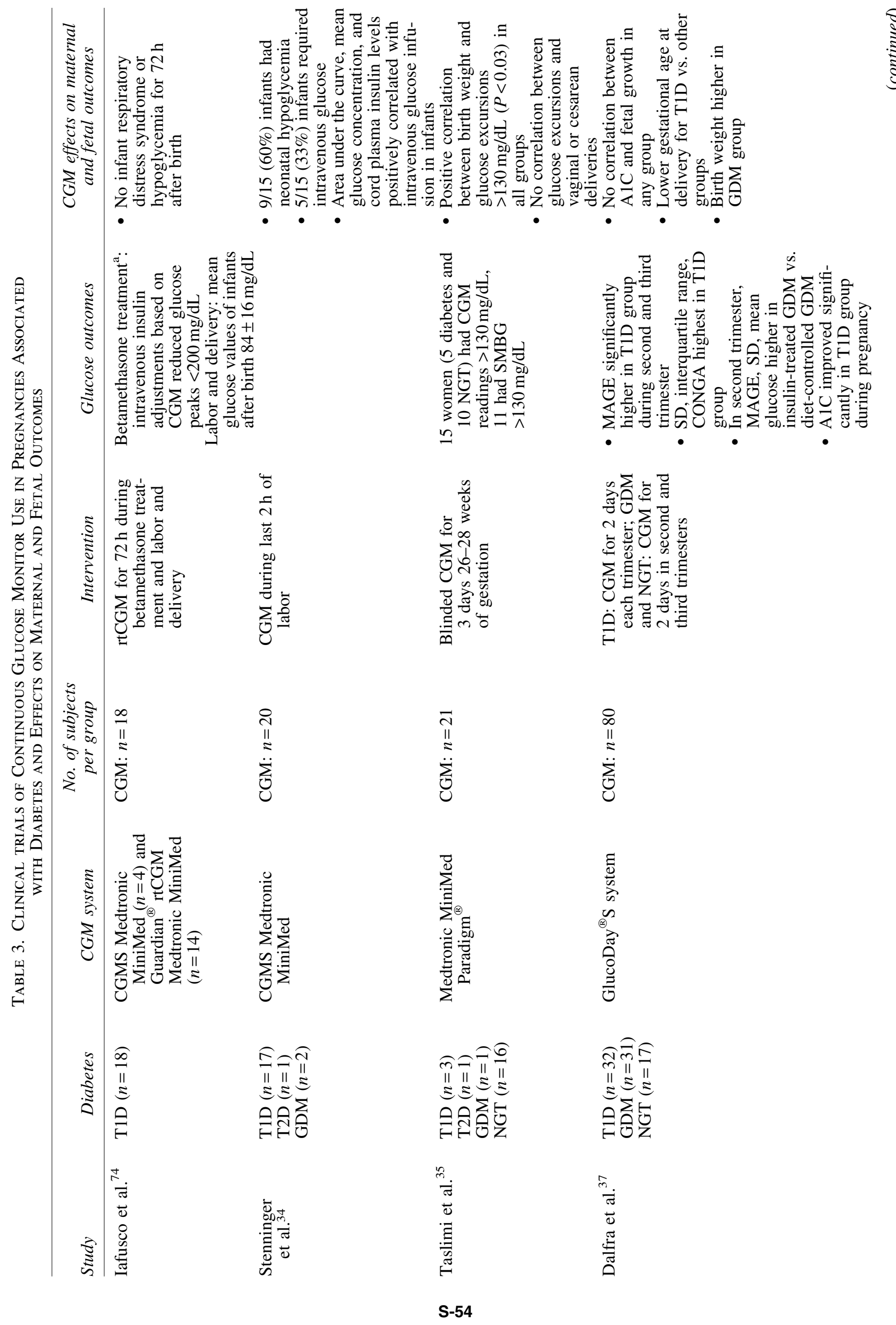




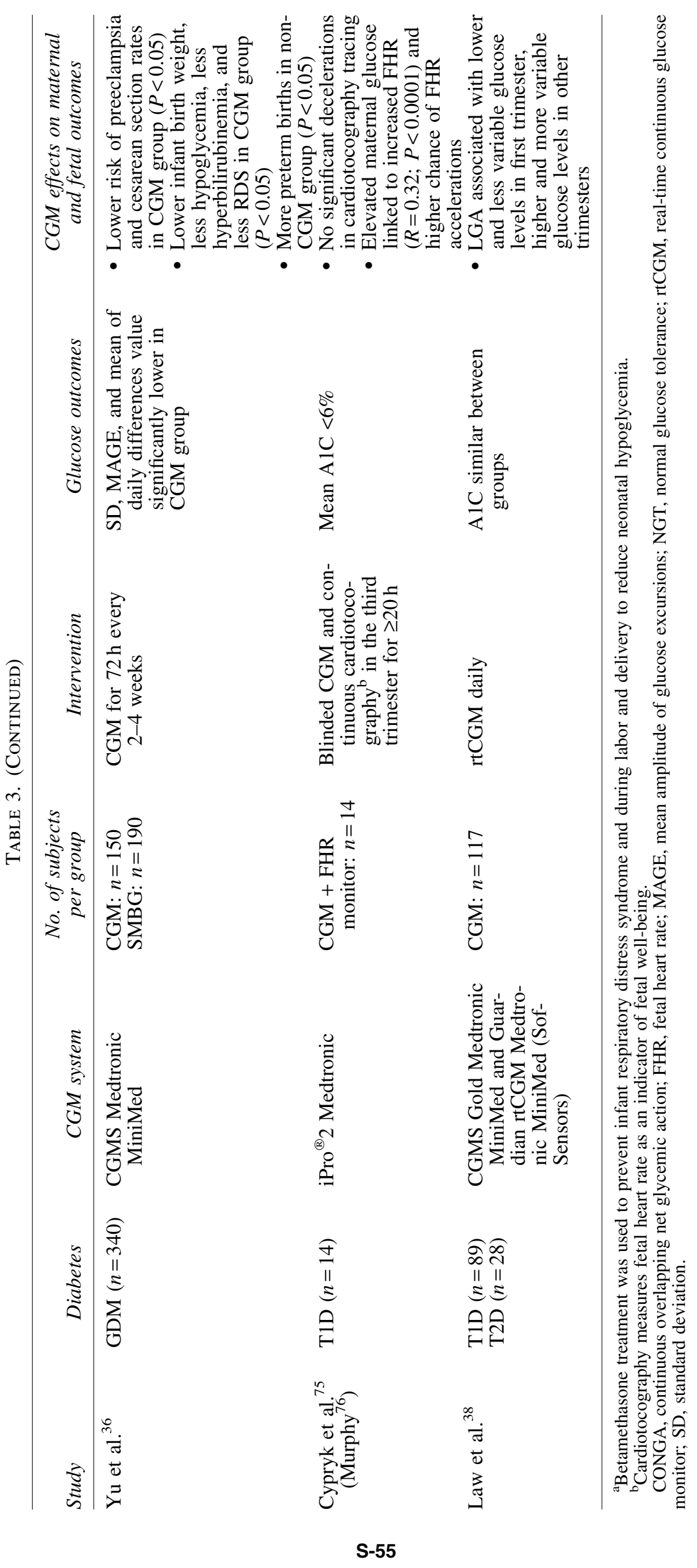


and 24-h glucose targets were significantly better in closedloop sessions with time in the target range (overnight: $74.7 \%$ closed-loop vs. $59.5 \%$ SAPT, $P=0.002 ; 24-$ h: $66.3 \%$ closedloop vs. $56.8 \%$ SAPT, $P<0.001$ ), hyperglycemic ranges, and mean glucose levels. After the crossover study, 14 women continued closed-loop therapy until delivery. The median glucose level $24 \mathrm{~h}$ before delivery was $110 \mathrm{mg} / \mathrm{dL}$ with $86.8 \%$ time in the target range. ${ }^{46}$ Of note, in supplementary material, it is reported that there were numerous device deficiencies during this study for the CGM, CSII, tablet computer, and downloading of devices, but none of them led to severe adverse glycemic or clinical outcomes. Thus, SAPT and closedloop insulin delivery systems need further evaluation to determine if they improve glucose control in pregnant women with diabetes.

\section{Caveats about CGM Use in Pregnancy}

CGM is beneficial as an adjunctive glucose management tool in pregnancy, but has limitations. Many limitations are inherent to the technology and apply in and outside of pregnancy. Barriers to successful use of CGM include discomfort, sensor accuracy, pharmacologic interference with readings, cost, reimbursement issues, and lack of time and training for healthcare professionals. ${ }^{47-49}$

Participants in the T1D Exchange clinic registry reported a discontinuation rate of $41 \%$ within a year of CGM initiation from device discomfort or technical problems. ${ }^{49}$ As the abdomen becomes more protuberant in pregnancy, comfortable sensor insertions may be challenging. Although other sites are considered off-label use, we and other practices have found that sensor insertions in the arm or flank are worth considering in the third trimester. Sensor accuracy can be a barrier despite recent improvements. ${ }^{47}$ Pharmacologic agents, such as acetaminophen, aspirin, and vitamin $\mathrm{C}$, can give falsely high or low sensor glucose readings. ${ }^{48}$ This is problematic in pregnancy as acetaminophen is the most compatible analgesic given that nonsteroidal anti-inflammatory drugs are contraindicated. Additionally, aspirin is becoming a common gestational therapy because when started by 16 weeks, the risk of preeclampsia is reduced in pregnancies complicated by diabetes. $^{50}$ CGM accuracy during hypoglycemia is questionable ${ }^{47}$ which is concerning given the increased hypoglycemia risk associated with tight glycemic pregnancy targets. ${ }^{51,52}$ Furthermore, CGM systems measure interstitial fluid glucose as a marker of blood glucose with a lag time. The lag (5$10 \mathrm{~min}$ ) is greatest during times of rapidly changing plasma glucose levels and can affect calibrations and long-term accuracy of the CGM. ${ }^{53}$ Another potential problem is the physiologic change in the interstitial fluid that accompanies pregnancy and may impact sensor accuracy, although one study found that this was not relevant for the sensor used. ${ }^{44}$

As with any medical device, CGMs can be costprohibitive. CGM coverage from payers has improved, but may still be expensive for patients. Diabetes in pregnancy requires frequent provider visits to obstetricians, endocrinologists, and other specialists. Adding costs to visit copays and prescriptions may not be feasible for some patients. Additional barriers are poor reimbursement and lack of training for healthcare professionals. ${ }^{47}$

CGM use in pregnancy is not a substitute for SMBG, but rather a supplemental means of capturing glucose levels and trends. ${ }^{7}$ SMBG measurements are often required for calibrations for CGM systems. Seven-point profiles and glucose $\log$ sheets are recommended for routine antenatal care in diabetic pregnancies. ${ }^{2}$ In women on MDIs, CGM does not provide information on insulin doses or carbohydrates consumed such as is seen on glucose log sheets, making insulin adjustments challenging based on CGM data alone. CGM technology has improved drastically since its introduction. ${ }^{47}$ As this continues, many barriers may recede, thus enhancing the utility of CGM therapy in pregnancy.

Finally, it is important to note that most of the studies included in this review have relatively small sample sizes. Thus, any conclusions that one can make, in particular regarding glycemic control and clinical outcomes, are limited. More and larger studies are warranted.

\section{Remote Monitoring in Pregnancy}

An emerging area of study is how the use of remote monitoring of glucose data affects patient outcomes. Remote monitoring includes the ability to see glucose meter data, CGM data as trends or daily patterns, and insulin pump downloads. Regarding CGM remote monitoring, some companies have software specifically for their products, such as CareLink and CareLink Pro for Medtronic products ${ }^{54-59}$ and Studio, Share, and Clarity for Dexcom products. ${ }^{60,61}$ Other programs can download multiple devices and view data in one system, such as Glooko, ${ }^{61-63}$ Tidepool Blip, ${ }^{61,64}$ and Diasend. $^{61,65}$ Software and online programs have been used in remote or retrospective monitoring of glucose data in nonpregnant populations. ${ }^{55-59,66-68}$

One CGM system was evaluated with concurrent remote monitoring at children's diabetes camps. On control nights of CGM use, subjects $(n=57)$ could hear hypoglycemia alarms, but on intervention nights, only medical personnel received alarms through remote monitoring. Subjects were evaluated under control and intervention conditions on alternating nights for each. The intervention significantly reduced the hypoglycemic events ( $<70 \mathrm{mg} / \mathrm{dL})$ lasting $1 \mathrm{~h}$ ( 7 intervention vs. 33 control, $P=0.003)$ and $2 \mathrm{~h}(0$ intervention vs. 12 control, $P=0.01)$, reduced extreme hypoglycemic events $(<50 \mathrm{mg} / \mathrm{dL})$ lasting $>30$ min ( 0 intervention vs. 9 control, $P=0.021)$, and improved response rates to CGM hypoglycemia (100\% intervention vs. $54 \%$ control arm). ${ }^{66}$

Some studies examined remote monitoring of glucose meter data by a provider who then adjusted treatment in pregnant women with GDM or T1D. ${ }^{69-72}$ We presented data at the American Diabetes Association (ADA) Scientific Sessions of interim analyses of a study using CGM alone or with Share ${ }^{\mathrm{TM}}$ remote monitoring technology in pregnant women with T1D. Share allows followers (family and friends) to view sensor glucose trends and receive alerts. There was a trend for improved glucose control and reduced fear of hypoglycemia with CGM Share use. ${ }^{73}$ More studies are needed to examine how remote monitoring affects glucose control and pregnancy outcomes.

\section{Conclusions}

CGM use appears to be safe and effective in pregnancies complicated by diabetes. CGMs can help identify glycemic patterns in pregnancy, obtain and maintain glucose targets, and reduce hypoglycemia. CGM helps with treatment adjustments 
in pregnancies associated with diabetes. It is unclear whether CGM use in pregnancy affects maternal and fetal outcomes. Using CSII in conjunction with rtCGM (SAPT) may improve outcomes in pregnancies associated with diabetes. CGM is not a substitute for standard clinical care, such as adhering to carbohydrate intake goals, collecting seven-point profiles, and filling in log sheets (MDI patients). Although more studies are needed, CGM use has promise as a therapy to improve outcomes in pregnancies associated with diabetes.

\section{Author Disclosure Statement}

Research funding was provided by DexCom, Inc., and National Institutes of Diabetes and Digestive Kidney Diseases.

\section{References}

1. Crowther CA, Hiller JE, Moss JR, et al.: Effect of treatment of gestational diabetes mellitus on pregnancy outcomes. N Engl J Med 2005;352:2477-2486.

2. Kitzmiller JL, Block JM, Brown FM, et al.: Managing preexisting diabetes for pregnancy: summary of evidence and consensus recommendations for care. Diabetes Care 2008;31:1060-1079.

3. Catalano PM, McIntyre HD, Cruickshank JK, et al.: The hyperglycemia and adverse pregnancy outcome study: associations of GDM and obesity with pregnancy outcomes. Diabetes Care 2012;35:780-786.

4. Jensen DM, Damm P, Moelsted-Pedersen L, et al.: Outcomes in type 1 diabetic pregnancies: a nationwide, populationbased study. Diabetes Care 2004;27:2819-2823.

5. American Diabetes Association. Management of diabetes in pregnancy. Sec. 12. In Standards of Medical Care in Diabetes - 2016. Diabetes Care 2016;39 Suppl 1:S94-S98.

6. Juvenile Diabetes Research Foundation Continuous Glucose Monitoring Study Group, Tamborlane WV, Beck RW, et al.: Continuous glucose monitoring and intensive treatment of type 1 diabetes. N Engl J Med 2008;359:1464-1476.

7. Pickup JC, Freeman SC, Sutton AJ: Glycaemic control in type 1 diabetes during real time continuous glucose monitoring compared with self monitoring of blood glucose: meta-analysis of randomised controlled trials using individual patient data. BMJ 2011;343:d3805.

8. Battelino T, Conget I, Olsen B, et al.: The use and efficacy of continuous glucose monitoring in type 1 diabetes treated with insulin pump therapy: a randomised controlled trial. Diabetologia 2012;55:3155-3162.

9. Chen R, Yogev Y, Ben-Haroush A, et al.: Continuous glucose monitoring for the evaluation and improved control of gestational diabetes mellitus. J Matern Fetal Neonatal Med 2003;14:256-260.

10. Yogev Y, Ben-Haroush A, Chen R, et al.: Continuous glucose monitoring for treatment adjustment in diabetic pregnancies - a pilot study. Diabet Med 2003;20:558-562.

11. Kerssen A, de Valk HW, Visser GH: The Continuous Glucose Monitoring System during pregnancy of women with type 1 diabetes mellitus: accuracy assessment. Diabetes Technol Ther 2004;6:645-651.

12. Secher AL, Stage E, Ringholm L, et al.: Real-time continuous glucose monitoring as a tool to prevent severe hypoglycaemia in selected pregnant women with Type 1 diabetes-an observational study. Diabet Med 2014;31:352-356.

13. Buhling KJ, Kurzidim B, Wolf C, et al.: Introductory experience with the continuous glucose monitoring system
(CGMS; Medtronic Minimed) in detecting hyperglycemia by comparing the self-monitoring of blood glucose (SMBG) in non-pregnant women and in pregnant women with impaired glucose tolerance and gestational diabetes. Exp Clin Endocrinol Diabetes 2004;112:556-560.

14. Yogev Y, Chen R, Ben-Haroush A, et al.: Continuous glucose monitoring for the evaluation of gravid women with type 1 diabetes mellitus. Obstet Gynecol 2003;101: 633-638.

15. Ben-Haroush A, Yogev Y, Chen R, et al.: The postprandial glucose profile in the diabetic pregnancy. Am J Obstet Gynecol 2004;191:576-581.

16. Kerssen A, de Valk HW, Visser GH: Day-to-day glucose variability during pregnancy in women with Type 1 diabetes mellitus: glucose profiles measured with the Continuous Glucose Monitoring System. BJOG 2004;111:919-924.

17. Kerssen A, Evers IM, de Valk HW, Visser GH: Poor glucose control in women with type 1 diabetes mellitus and 'safe' hemoglobin A1c values in the first trimester of pregnancy. J Matern Fetal Neonatal Med 2003;13:309-313.

18. Kerssen A, de Valk HW, Visser GH: Do HbA1c levels and the self-monitoring of blood glucose levels adequately reflect glycaemic control during pregnancy in women with type 1 diabetes mellitus? Diabetologia 2006;49:25-28.

19. Buhling KJ, Winkel T, Wolf C, et al.: Optimal timing for postprandial glucose measurement in pregnant women with diabetes and a non-diabetic pregnant population evaluated by the Continuous Glucose Monitoring System (CGMS). J Perinat Med 2005;33:125-131.

20. Tonoike M, Kishimoto M, Yamamoto M, et al.: Continuous glucose monitoring in patients with abnormal glucose tolerance during pregnancy: A case series. Jpn Clin Med 2016; 7:1-8.

21. Mazze R, Yogev Y, Langer O: Measuring glucose exposure and variability using continuous glucose monitoring in normal and abnormal glucose metabolism in pregnancy. J Matern Fetal Neonatal Med 2012;25:1171-1175.

22. Dalfra MG, Chilelli NC, Di Cianni G, et al.: Glucose fluctuations during gestation: an additional tool for monitoring pregnancy complicated by diabetes. Int J Endocrinol 2013;2013:279021.

23. Bonis C, Lorenzini F, Bertrand M, et al.: Glucose profiles in pregnant women after a gastric bypass: findings from continuous glucose monitoring. Obes Surg 2016;26:2150-2155.

24. Harmon KA, Gerard L, Jensen DR, et al.: Continuous glucose profiles in obese and normal-weight pregnant women on a controlled diet: metabolic determinants of fetal growth. Diabetes Care 2011;34:2198-2204.

25. Naik D, Asha HS, Mruthyunjaya MD, et al.: Masked hypoglycemia in pregnancy. J Diabetes 2016 [Epub ahead of print]; DOI: 10.1111/1753-0407.12485.

26. Kumareswaran K, Elleri D, Allen JM, et al.: Accuracy of continuous glucose monitoring during exercise in type 1 diabetes pregnancy. Diabetes Technol Ther 2013;15:223-229.

27. Kestila KK, Ekblad UU, Ronnemaa T: Continuous glucose monitoring versus self-monitoring of blood glucose in the treatment of gestational diabetes mellitus. Diabetes Res Clin Pract 2007;77:174-179.

28. Murphy HR, Rayman G, Lewis K, et al.: Effectiveness of continuous glucose monitoring in pregnant women with diabetes: randomised clinical trial. BMJ 2008;337:a1680.

29. Murphy HR, Rayman G, Duffield K, et al.: Changes in the glycemic profiles of women with type 1 and type 2 diabetes during pregnancy. Diabetes Care 2007;30:2785-2791. 
30. Cordua S, Secher AL, Ringholm L, et al.: Real-time continuous glucose monitoring during labour and delivery in women with Type 1 diabetes-observations from a randomized controlled trial. Diabet Med 2013;30:1374-1381.

31. Secher AL, Ringholm L, Andersen HU, et al.: The effect of real-time continuous glucose monitoring in pregnant women with diabetes: a randomized controlled trial. Diabetes Care 2013;36:1877-1883.

32. Feig DS, Asztalos E, Corcoy R, et al.: CONCEPTT: continuous glucose monitoring in women with type 1 diabetes in pregnancy trial: a multi-center, multi-national, randomized controlled trial-study protocol. BMC Pregnancy Childbirth 2016;16:167.

33. Voormolen DN, DeVries JH, Franx A, et al.: Effectiveness of continuous glucose monitoring during diabetic pregnancy (GlucoMOMS trial); a randomised controlled trial. BMC Pregnancy Childbirth 2012;12:164.

34. Stenninger E, Lindqvist A, Aman J, et al.: Continuous subcutaneous glucose monitoring system in diabetic mothers during labour and postnatal glucose adaptation of their infants. Diabet Med 2008;25:450-454.

35. Taslimi MM, Navabi K, Acosta R, et al.: Concealed maternal blood glucose excursions correlate with birth weight centile. J Diabetes Sci Technol 2008;2:456-460.

36. Yu F, Lv L, Liang Z, et al.: Continuous glucose monitoring effects on maternal glycemic control and pregnancy outcomes in patients with gestational diabetes mellitus: a prospective cohort study. J Clin Endocrinol Metab 2014;99: 4674-4682.

37. Dalfra MG, Sartore G, Di Cianni G, et al.: Glucose variability in diabetic pregnancy. Diabetes Technol Ther 2011; 13:853-859.

38. Law GR, Ellison GT, Secher AL, et al.: Analysis of continuous glucose monitoring in pregnant women with diabetes: Distinct temporal patterns of glucose associated with large-for-gestational-age infants. Diabetes Care 2015;38: 1319-1325.

39. Davis SN, Horton ES, Battelino T, et al.: STAR 3 randomized controlled trial to compare sensor-augmented insulin pump therapy with multiple daily injections in the treatment of type 1 diabetes: research design, methods, and baseline characteristics of enrolled subjects. Diabetes Technol Ther 2010;12:249-255.

40. Bergenstal RM, Tamborlane WV, Ahmann A, et al.: Effectiveness of sensor-augmented insulin-pump therapy in type 1 diabetes. N Engl J Med 2010;363:311-320.

41. Raccah D, Sulmont V, Reznik Y, et al.: Incremental value of continuous glucose monitoring when starting pump therapy in patients with poorly controlled type 1 diabetes: the RealTrend study. Diabetes Care 2009;32: 2245-2250.

42. Fresa R, Visalli N, Di Blasi V, et al.: Experiences of continuous subcutaneous insulin infusion in pregnant women with type 1 diabetes during delivery from four Italian centers: a retrospective observational study. Diabetes Technol Ther 2013;15:328-334.

43. Petrovski G, Dimitrovski C, Bogoev M, et al.: Is there a difference in pregnancy and glycemic outcome in patients with type 1 diabetes on insulin pump with constant or intermittent glucose monitoring? A pilot study. Diabetes Technol Ther 2011;13:1109-1113.

44. Murphy HR, Elleri D, Allen JM, et al.: Closed-loop insulin delivery during pregnancy complicated by type 1 diabetes. Diabetes Care 2011;34:406-411.
45. Murphy HR, Kumareswaran K, Elleri D, et al.: Safety and efficacy of 24-h closed-loop insulin delivery in wellcontrolled pregnant women with type 1 diabetes: a randomized crossover case series. Diabetes Care 2011;34: 2527-2529.

46. Stewart ZA, Wilinska ME, Hartnell S, et al.: Closed-loop insulin delivery during pregnancy in women with type 1 diabetes. N Engl J Med 2016;375:644-654.

47. Rodbard D: Continuous glucose monitoring: a review of successes, challenges, and opportunities. Diabetes Technol Ther 2016;18 Suppl 2:S23-S213.

48. Basu A, Veettil S, Dyer R, et al.: Direct evidence of acetaminophen interference with subcutaneous glucose sensing in humans: a pilot study. Diabetes Technol Ther 2016;18 Suppl 2:S243-S247.

49. Anhalt $\mathrm{H}$ : Limitations of continuous glucose monitor usage. Diabetes Technol Ther 2016;18:115-117.

50. Roberge S, Nicolaides $K$, Demers $S$, et al.: The role of aspirin dose on the prevention of preeclampsia and fetal growth restriction: systematic review and meta-analysis. Am J Obstet Gynecol 2016.

51. Evers IM, ter Braak EW, de Valk HW, et al.: Risk indicators predictive for severe hypoglycemia during the first trimester of type 1 diabetic pregnancy. Diabetes Care 2002;25:554-559.

52. Nielsen LR, Pedersen-Bjergaard U, Thorsteinsson B, et al.: Hypoglycemia in pregnant women with type 1 diabetes: predictors and role of metabolic control. Diabetes Care 2008;31:9-14.

53. Cengiz E, Tamborlane WV: A tale of two compartments: interstitial versus blood glucose monitoring. Diabetes Technol Ther 2009;11 Suppl 1:S11-S16.

54. Welsh JB, Myers SJ, Uhrinak AN, et al.: User acceptability and perceived benefits of new reports in CareLink Pro 3.0 Therapy Management Software for Diabetes. J Diabetes Sci Technol 2012;6:481-482.

55. Quiros C, Patrascioiu I, Gimenez M, et al.: Assessment of use of specific features of subcutaneous insulin infusion systems and their relationship to metabolic control in patients with type 1 diabetes. Endocrinol Nutr 2014;61:318322.

56. Shalitin S, Ben-Ari T, Yackobovitch-Gavan M, et al.: Using the Internet-based upload blood glucose monitoring and therapy management system in patients with type 1 diabetes. Acta Diabetol 2014;51:247-256.

57. Battelino T, Liabat S, Veeze HJ, et al.: Routine use of continuous glucose monitoring in 10501 people with diabetes mellitus. Diabet Med 2015;32:1568-1574.

58. Agrawal P, Zhong A, Welsh JB, et al.: Retrospective analysis of the real-world use of the threshold suspend feature of sensor-augmented insulin pumps. Diabetes Technol Ther 2015;17:316-319.

59. Zhong A, Choudhary P, McMahon C, et al.: Effectiveness of automated insulin management features of the Mini$\operatorname{Med}(\mathrm{R})$ 640G sensor-augmented insulin pump. Diabetes Technol Ther 2016;18:657-663.

60. Dexcom Clarity. 2017. https://www.dexcom.com/clarity (accessed May 5, 2017).

61. Hoskins M: Finding CLARITY in Diabetes Data Analysis (Thanks, Dexcom!). September 22, 2015; www.healthline .com/diabetesmine/finding-clarity-diabetes-data-displaysthanks-dexcom\#1 (accessed December 27, 2016).

62. glooko 2016. https://www.glooko.com/providers-payers (accessed December 27, 2016). 
63. Hoskins M: Glooko Brings Mobile Diabetes Data-Sharing to Pumps and CGM Devices. 2016. August 19, 2015. www .healthline.com/diabetesmine/glooko-diabetes-data-sharingupdate\#1 (accessed December 28, 2016).

64. Neinstein A, Wong J, Look H, et al.: A case study in open source innovation: developing the Tidepool Platform for interoperability in type 1 diabetes management. J Am Med Inform Assoc 2016;23:324-332.

65. diasend Personal. 2016. https://www.diasend.com/us/patient (accessed December 28, 2016).

66. DeSalvo DJ, Keith-Hynes P, Peyser T, et al.: Remote glucose monitoring in cAMP setting reduces the risk of prolonged nocturnal hypoglycemia. Diabetes Technol Ther 2014;16:1-7.

67. Ng SM: Improving patient outcomes with technology and social media in paediatric diabetes. BMJ Qual Improv Rep 2015;4.

68. Barnard K, James J, Kerr D, et al.: Impact of chronic sleep disturbance for people living with $\mathrm{t} 1$ diabetes. J Diabetes Sci Technol 2016;10:762-767.

69. Homko CJ, Santamore WP, Whiteman V, et al.: Use of an internet-based telemedicine system to manage underserved women with gestational diabetes mellitus. Diabetes Technol Ther 2007;9:297-306.

70. Hirst JE, Mackillop L, Loerup L, et al.: Acceptability and user satisfaction of a smartphone-based, interactive blood glucose management system in women with gestational diabetes mellitus. J Diabetes Sci Technol 2015;9:111-115.

71. Perez-Ferre N, Galindo M, Fernandez MD, et al.: The outcomes of gestational diabetes mellitus after a telecare approach are not inferior to traditional outpatient clinic visits. Int J Endocrinol 2010;2010:386941.
72. Wojcicki JM, Ladyzynski P, Krzymien J, et al.: What we can really expect from telemedicine in intensive diabetes treatment: results from 3-year study on type 1 pregnant diabetic women. Diabetes Technol Ther 2001;3:581-589.

73. Polsky S, Voelmle M, Joshee P, et al.: Improved Glucose Control in Pregnant Women with Type 1 Diabetes Using Continuous Glucose Monitoring (CGM) Share System. Paper presented at: American Diabetes Association 76th Scientific Sessions; June 12, 2016; New Orleans, LA.

74. Iafusco D, Stoppoloni F, Salvia G, et al.: Use of real time continuous glucose monitoring and intravenous insulin in type 1 diabetic mothers to prevent respiratory distress and hypoglycaemia in infants. BMC Pregnancy Childbirth 2008;8:23.

75. Cypryk K, Bartyzel L, Zurawska-Klis M, et al.: Continuous glucose monitoring in type 1 diabetes pregnancy shows that fetal heart rate correlates with maternal glycemia. Diabetes Technol Ther 2015;17:619-624.

76. Murphy HR: Maternal glycemia and fetal well-being: continuous glucose monitoring and continuous cardiotocography. Diabetes Technol Ther 2015;17:603-604.

Address correspondence to:

Sarit Polsky, MD, MPH

Barbara Davis Center for Diabetes University of Colorado Denver 1775 Aurora Court, MS A140 Aurora, CO 80045

E-mail: sarit.polsky@ucdenver.edu 